\title{
五种鲟鱼线粒体控制区异质性和系统发育分析
}

\author{
王 巍, 朱 华, 胡红霞, 田照辉, 董 颖 \\ (国家淡水渔业工程技术研究中心暨北京市水产科学研究所, 北京 100068)
}

\begin{abstract}
摘要: 利用保守引物得到 5 种鲟鱼的线粒体 DNA（mtDNA）控制区（D-loop）全长，长度在 795 813bp。序 列中包括了 CBS（conserved sequence block）和 TAS（termination-associated sequence）区域。利用最大似然法、 最大简约法和贝叶斯法构建了系统发育树, 发育树分成两枝, 呈现明显的生物地理分布。分析表明, 现有的鳇属 鱼类不是单系群起源。 5 种鲟鱼 D-loop 序列都存在长度和数目不等串联重复序列, 长度在 78 82bp 之间, 重复序 列拷贝数在 4 6 次不等, 因此造成了 mtDNA 广泛的异质性现象。不同种类的重复序列单元十分相似, 达氏鳇和 史氏鲄重复序列单元相似度为 $82.93 \%$, 西伯利亚鲟和俄罗斯鲟重复序列单元相似度为 $90.59 \%$ 。在串联重复序列 后是一段不完全重复序列。通过与已有同种的重复序列比对发现不同鲟鱼重复序列相同，不同地理区域相同物种 的重复序列可能发生过分子内重组。这些表明重复序列在鲟鱼进化上具有相关意义, 推测重复序列可能产生在种 分化前, 重组发生在种分化后。
\end{abstract}

关键词：线粒体控制区；西伯利亚鲟；史氏鲟；小体鲟；达氏鲌；俄罗斯鲟；重复序列；异质性

中图分类号: Q349.5; Q959.463 文献标识码: A 文章编号: 0254-5853-(2009)05-0487-010

\section{Heteroplasmy in mtDNA Control Region and Phylogenetics of Five Sturgeons}

\author{
WANG Wei, ZHU Hua, HU Hong-xia*, TIAN ZHAO-hui, DONG Ying \\ (National Engineering Research Center for Freshwater Fisheries and Beijing Fisheries Research Institute, Beijing 100068, China)
}

\begin{abstract}
The sequences of the control region (D-loop) of mitochondrial DNA ( mtDNA) were obtained from five species of sturgeon. The sequences obtained had lengths between 795-813bp. The motifs of the conserved sequence block (CBS) and termination-associated sequence (TAS) were identified in the sequences. Examination of the molecular phylogeny using maximum likelihood, maximum parsimony and Bayesian analysis shows that sturgeons form two clades representing biogeographic distribution, and the Huso is not monophyletic. Heteroplasmy in 150 fishes was due to a variable number of tandem repeats. Length of the central repeat units ranges from 78 to $82 \mathrm{bp}$ with repeat sequence copies between four and six. The repeat units, followed by an imperfect repeat unit, are in high similarity between species, with 82.93\% between Amur sturgeon and starlet and 90.59\% between Siberian sturgeon and Russian sturgeon. Furthermore, comparing with other repeat sequences published, different species have the same repeat unit and intramolecular recombination event may happen in the repeat unit. All of these indicate that the repeat units are related to the evolution of sturgeon and arose prior to species divergence after which recombination happened.
\end{abstract}

Key words: Mitochondrial control region; Acipenser baerii; A. schrenckii; A. ruthenus; Huso dauricus; A. gueldenstaedtii; Tandem repeat; Heteroplasmy

具有 “活化石”之称的鲟形目 (Acipenseriformes) 是一类古老的软骨硬鳞鱼类, 起源于泥盆纪（距今 $4.5 \sim 3.5$ 亿年前) (Gardiner, 1984 ), 对研究脊椎动物进化有重要意义。现存大 约有 27 种鲟鱼隶属于鲟科 (Acipenseridae) 和白鲟
科 (Polyodontidae), 其中鲟科, 占绝大多数, 有 4 属约 25 种; 而白鲟科仅有 2 属, 共 2 种 (Billard \& Lecointre, 2000; Ludwig, 2006)。它们主要分布于北 半球, 具有重要的经济价值（Bemis \& Findeis, 1994)。近些年来, 由于过度捕捞、水环境污染和 
大型水利工程的兴建等原因导致鲟鱼栖息环境破 坏严重, 野生种群减少, 并且鲟鱼本身性成熟晚, 幼体成活率低, 种群恢复慢, 使其在全球范围内均 处于不同程度的濒危状态, 已被列为《濒危动植物 种国际贸易公约》附录 II 物种 (Bemis \& Kynard, 1997)。

线粒体DNA排列紧密、进化速率快和几乎完全 母系遗传的特征, 使其广泛用于进化研究和种群遗 传分析 (Brown et al, 1979)。线粒体DNA不同区域 的进化速率存在差异, 其中控制区序列（D-loop） 位于 $\mathrm{tRNA}^{\mathrm{pro}}$ 和 $\mathrm{tRNA}^{\mathrm{ph}}$ 之间, 是线粒体双链转录启 动子和 $\mathrm{H}$ 链复制起始点所在区域 (Clayton, 1982; Walberg \& Clayton, 1981), 受到选择压力比mtDNA 其它区域要小, 为线粒体基因组中碱基突变和长度 变化最大的区域, 进化速率最快（Brown, 1985), 因而较适合用于种群遗传结构分析和系统发育分 析 (Boyce et al, 1989; Quattro et al, 2002; Zhang et al, 2003; Ludwig et al, 2008)。本研究对人工养殖的 5 种鲟鱼西伯利亚鲟 (Acipenser baerii)、俄罗斯鲟 ( $A$. gueldenstaedtii)、小体鲟 (A. ruthenus)、史氏鲟 (A. schrenckii）和达氏鳇（Huso dauricus）的线粒体控 制区序列进行了分析, 拟为鲟鱼育种提供遗传背景 分析依据。

\section{1 材料和方法}

\section{1 材 料}

鲟鱼样本取自北京市水产科学研究所十渡鲟 鱼养殖基地。西伯利亚鲟、俄罗斯鲟、小体鲟、史 氏鲟和达氏鲌各 30 条, 其中西伯利亚鲟、俄罗斯 鲟、小体鲟为 1996-2000 年从欧洲引进的人工繁 殖鱼苗, 史氏鲟和达氏鳇为 1999-2001 年捕捞于 黑龙江的野生亲鱼人工繁殖后代。除部分达氏鳇雌 鱼外, 所有的鲟鱼均已性腺发育成熟。剪取部分鯺 条, 保存于 95\%酒精中。

\section{2 总 DNA 提取和 mtDNA 控制区的扩增}

剪取约 $0.5 \mathrm{~g}$ 的鲟鱼鯺条样本, 加 $1.0 \mathrm{~mL}$ 组织
匀浆液 $(100 \mathrm{mmol} / \mathrm{L} \mathrm{NaCl} ; 10 \mathrm{mmol} / \mathrm{L}$ Tris- $\mathrm{HCl}$, $\mathrm{pH}$ 8.0； $0.25 \mathrm{mmol} / \mathrm{L}$ EDTA, pH 8.0）混合匀浆, 然 后加 $0.4 \mathrm{~mL}$ 酶解液 $(200 \mathrm{mmol} / \mathrm{L} \mathrm{NaCl} ; 10 \mathrm{mmol} / \mathrm{L}$ Tris-HCl pH 8.0; 50 mmol/L EDTA, pH 8.0; 200 $\mu \mathrm{g} / \mathrm{mL}$ 蛋白酶 $\mathrm{K} ; 1 \% \mathrm{SDS}$ ）混匀, $55^{\circ} \mathrm{C}$ 水浴过夜, 次日利用酚氯仿抽提法提取总 DNA, 并用 TE 缓冲 液溶解, $4^{\circ} \mathrm{C}$ 保存。

从GenBank查到 7 种鲟形目鱼类线粒体基因组 序列 (gi: 25057128、25057438、30065662、38638375、 42632215、45387959、46135658), 经Clustal X 1.83 比对 (Thompson et al, 1997), 发现了 tRNA ${ }^{\text {pro 和 }}$

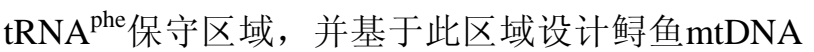
控制区扩增引物。D-loop全长用 1\%琼脂糖凝胶（1 $\times \mathrm{TAE})$ 电泳分离（120 V, 40 min), gel-red染色。 从 5 种鲟鱼基因组各取一个样本作为模板扩增出 D-loop全长序列, 连接pGEM-T载体 (Promega公 司), 转化培养后每个样本挑取 3 4 个克隆进行测 序, 以验证引物的可靠性。测序结果显示D-loop具 有重复序列片段。随后利用重复序列外的保守区域 设计特异性引物, 并对 5 个种 150 个样本进行扩增, PCR产物用 $1.5 \%$ 琼脂糖凝胶电泳 $(140 \mathrm{~V}$, 约 90 $\min$ ) 进行分析。引物序列分别为 tpro-f1： $5^{\prime}$ -AACTCCCAAAGCTAAGATTC-3' ; tphe-r2: 5' -ATGCTTTAGTTAAGCTACGC-3' ; Heteror3 : 5'-ATAGGAACCAGATGCCAGTA-3', 其中引物对 tpro-f1/tphe-r2 扩增 D-loop 序列全长, 引物对 tpro-f1/Hetero-r3 扩增D-loop重复序列区（图 1)。 $\mathrm{PCR}$ 反应体系 $(50 \mu \mathrm{L})$ 为: DNA 模板 $30 \mathrm{ng}, 6$ $\mathrm{mmol} / \mathrm{L} \mathrm{MgCl}_{2}, 1 \times$ 扩增缓冲液, 4 种 dNTP各 0.45 $\mathrm{mmol} / \mathrm{L}$ ，上下游引物各 $4.5 \mathrm{pmol} / \mathrm{L}, 1 \mathrm{U} \mathrm{r}-\mathrm{taq} \mathrm{DNA}$ 聚合酶。扩增全长和重复序列反应程序为: $95^{\circ} \mathrm{C}$ 预 变性 $4 \mathrm{~min} ; 95^{\circ} \mathrm{C} 45 \mathrm{~s}, 54^{\circ} \mathrm{C} 45 \mathrm{~s}, 72^{\circ} \mathrm{C} 90 \mathrm{~s}, 36$ 个循环; $72^{\circ} \mathrm{C}$ 最终延伸 $8 \mathrm{~min}$ 。

\section{3 序列的比对、遗传多样性及系统发生关系的分} 析

测序结果经人工校对拼接, 得到完整 D-loop

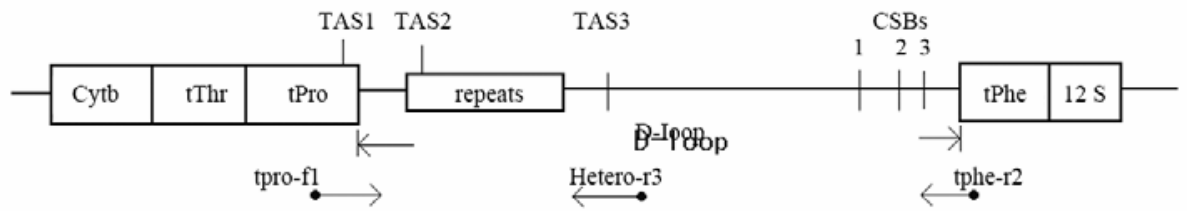

图 1 鲟鱼 mtDNA 控制区结构及 PCR 扩增示意图

Fig. 1 Schematic diagram of the D-loop structure and the location of the primers for PCR amplification 
序列。利用 Clustal X 1.83 (Thompson et al, 1997) 对所得到的序列进行完全比对分析, 同时与 GenBank 中的 D-loop 序列也进行比对, 确定得到的 是鲟鱼线粒体控制区序列。利用分子进化遗传分 析软件 PHYML 构建 ML 系统发育树 (Guindon \& Gascuel, 2003), 并设置 bootstrap 重复分析 1000 次

(Felsenstein, 1985 )。 Modeltest 3.7 ( Posada \& Crandall, 1998) 计算得到构建 ML 树最适模型为 $\mathrm{TrN}+\mathrm{I}+\mathrm{G}$ 。用 PAUP 4.0b4a（Swofford, 1998）构建 MP 系统发育树, 进行内部分支检验和 1000 次 bootstrap 重复分析 (Felsenstein, 1985 )。利用 MrBayes 3.1.2 (Ronquist \& Huelsenbeck, 2003) 构 建 Bayes 树, Mr modeltest 2.3 (Nylander, 2004) 计 算得到构建 Bayes 树最适模型为 GTR $+\mathrm{I}+\mathrm{G}$, 然后建 立 4 个马尔可夫链, 以随机树为起始树, 共运行 100 万代, 每 100 代抽样一次, 在舍弃老化样本后 (1000 代), 根据剩余样本构建一致树, 系统树各分支的 置信度评估用后验概率 (posterior probability value) 表示。利用 MFOLD 预测重复序列的二级结构, 并 计算热稳定值 $\triangle \mathrm{G}$ (Walter et al, 1994; Zuker, 1989)。

\section{2 结 果}

\section{1 五种鲟鱼 mtDNA 长度多态性}

经克隆测序后共得到 5 种鲟鱼D-loop全长及部 分编码 $\mathrm{tRNA}^{\text {pro }}$ 和 $\mathrm{tRNA}{ }^{\text {phe }}$ 序列, 序列已提交至 GenBank（gi: 226452516-20)。5 种鲟鱼的D-loop序 列长短不一, 出现不同长度的碱基插入/缺失, $\mathrm{AT}$ 含量达 $60 \%$ 以上 (表 1 ), 同时发现在靠近 $\mathrm{RNA}^{\mathrm{pro}}$ 端 存在不同程度重复的序列片段 (图 2)。对重复区域 的扩增产物电泳 (1.5\%琼脂糖凝胶) 检测后 150 个 个体全部出现多条条带, 其中达氏鳇、小体鲟和史 氏鲟的条带类型只有一种, 而俄罗斯鲟和西伯利亚 鲟分别有 2 种和 3 种条带类型, 且重复片段在序列 中至少重复 4 次 (如表 1 和图 3 所示)。

西伯利亚鲟、达氏鳇、俄罗斯鲟、小体鲟同一 样本 D-loop 的重复序列单元间无变异发生, 但在西 史杂交鲟 (史氏鲟为母本) 与史氏鲟间, 重复单元 间有一个变异位点, 发生在第一个重复单元第 32 位上 T-C, 其它重复单元序列是一致的 (未列出)。

\subsection{5 种鲟鱼系统发育}

用 GeneBank 中已有的 5 种鲟形目鱼类欧洲鳇 (Huso huso, gi: 38638375)、达氏鲟 (Acipenser dabryanus, gi: 42632215)、高首鲟 (A. transmontanus, gi: 30065662 ) 、匙吻鲟 (Polyodon spathula gi: 41387097) 和白鲟 (Psephurus gladius, gi: 45593284) 的 D-loop 序列与实验得到的 5 条序列进行比对, 并 构建系统发育树 (图 4)。从图 4 所得到的系统发育 树可以看出, 构建的 MP、ML、Bayes 树都具有相 同的拓扑结构, 都得到了较高的自展检验值和后验 概率。

\section{3 讨 论}

在许多脊椎动物均发现线粒体控制区序列存 在保守区域CBS (conserved sequence block) 和相对 保守区域 TAS (termination-associated sequence)

(Brown et al, 1986; Clayton, 1982)。CBS 位于 D-loop序列 3' 端, 被认为是线粒体DNA重链合成起 始时形成RNA引物的加工信号 (Clayton, 1984)。在 鲟鱼mtDNA中存在 3 个CBS, 分别为CBS1、CBS2、 CBS3, 它们的排列顺序十分保守 (图 2)。CBS1 位 于新 $\mathrm{H}$ 链上游, 一般被认为与将近完成 $\mathrm{H}$ 链替代合 成的终点信号有关; CBS2 和CBS3 位于D-loop 5' 端, 可能与 $\mathrm{H}$ 链的复制起始有关 (Walberg \& Clayton, 1981 ), 也是转录启动子所在区域 (Hauswirth \& Clayton, 1985)。CBS的核酸序列也很保守 (图 2), 大部分鱼类CBS1 具有 “GACATA” 结构, CBS2 和 CBS3 富含AC碱基（Broughton \& Dowling, 1994; Chen et al, 2004; Lee et al, 1995)。TAS区域靠近 tRNA ${ }^{\text {pro }}$, 位于D-loop序列 5' 端, 是线粒体DNA合 成终止时的信号 (Clayton, 1984), 其中TAS1 位于 tRNApro序列中, TAS2 在D-loop重复序列中, TAS1 与TAS2 的差异在 1 2 个碱基, 其保守性不及CBS (图 2)。

\section{1 系统发育分析}

从构建的系统发育树看，白鲟科 (Polyodontidae) 和鲟科 (Acipenseridae) 自展值 和后验概率都为 $100 \%$, 表明两者互为姊妹群。白 鲟科的白鲟和匙吻鲟进化距离较近, 与 Zhang et al （2000）根据 12 种鲟鱼 ND4L-ND4 基因构建的系 统发育树一致。此外, 利用形态学特征构建的系统 发育树也表明两者的距离相近 (Artyukhin, 2006)。 鲟科鱼类则分成两个分支, 呈现出明显的生物地理 分布, 分别被称做大西洋种和太平洋种 (Krieger et al, 2008), 其中西伯利亚鲟、俄罗斯鲟、小体鲟和 欧洲鳇聚在一起, 为大西洋种分支, 达氏鳇、达氏 鲟、史氏鲟和高首鲟分在一支, 为太平洋 
$1 \rightarrow T A S T \leftarrow 1$ TRA $1 \rightarrow \mathrm{D}-\mathrm{IOOP}$

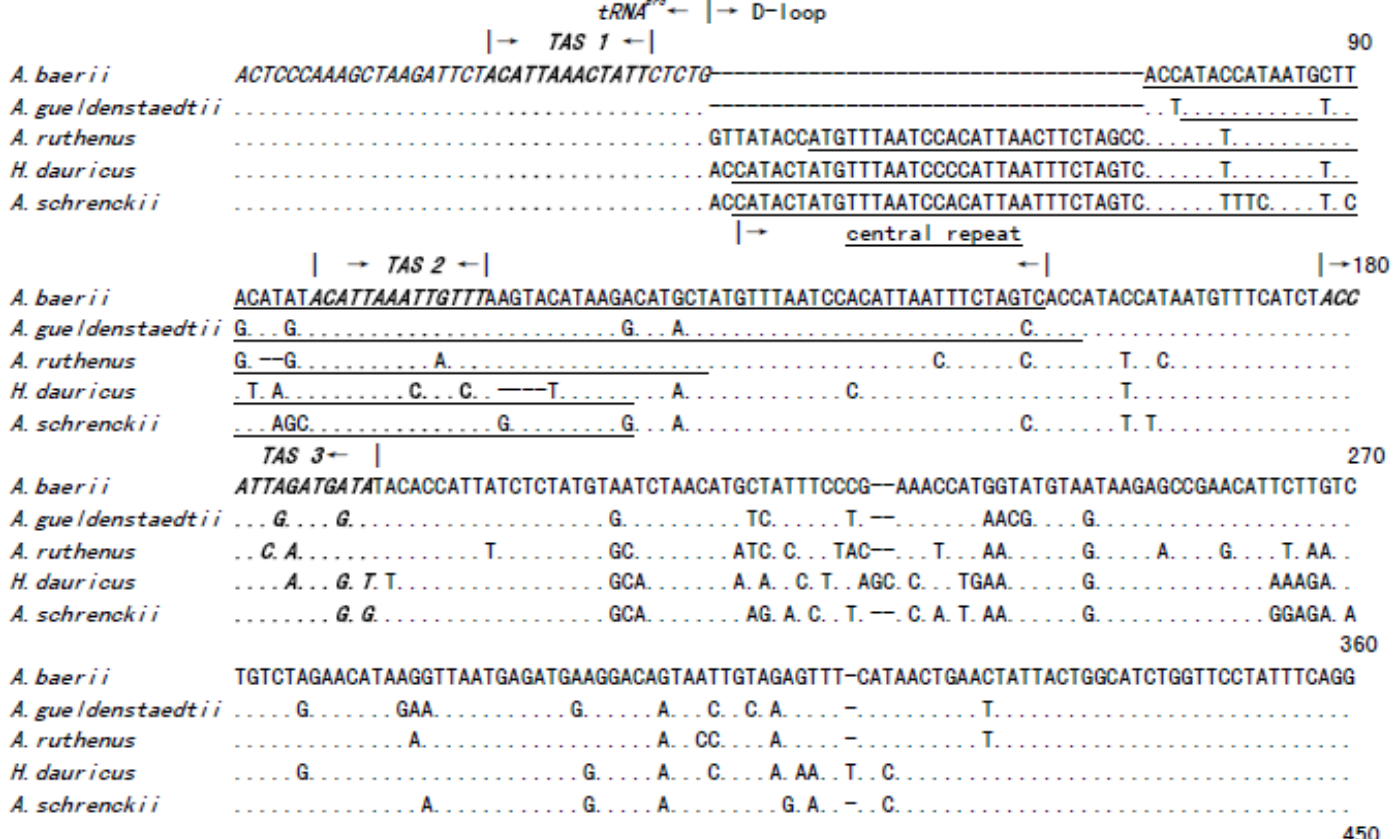

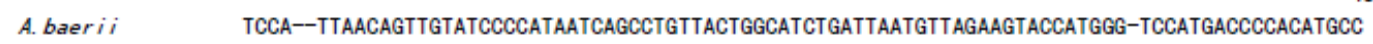

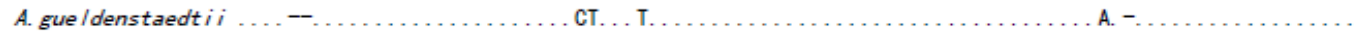

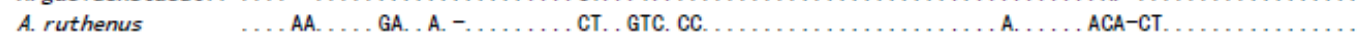

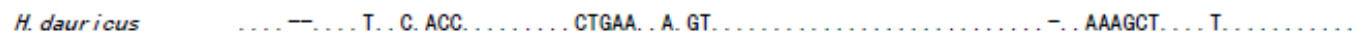

A. schrenckii $\quad$ C. . - . G. . G. . A. T. . . . . . CTGAA. . A. GT . . . . . . . G. . . G. G. . . T. AAAA . G. . . . . . .

A. baer i i GAGAACCCCATCAACATTTGGTATTTTTATTTTTAGGTCT-CCATTCACTGACATGCAGG-GCTCCTTCAGAAAGATAGACAGGGGG

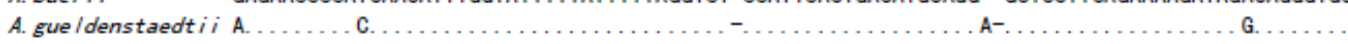

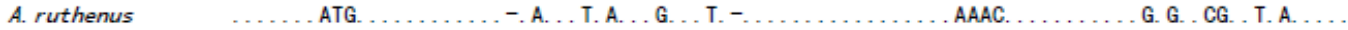
H. dauricus $\quad \ldots$. T. TTGC. . . . . . . . . . . . . . . . . . . . . . . AA- . . . . . . ACA. T. A. . . A. schrenckii $\quad$ A. . . T. TTG. . . . . . . . . . . . . . . . . . . . . . . . . . AA- . . . . . . ACA . A. .

A. baeri $i$ AACATTCACGAC-TGCTCAGAGATAATGAATAG TGAATGATATAATGACATACTCTGGT-ATACTACATGGTCTGTGCCACGTACATAAG

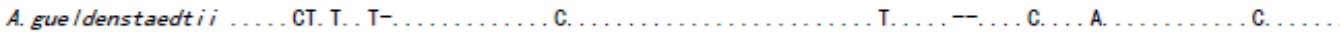
A. ruthenus $\quad \ldots$. . . GTC. . . G. . . CG. . G. . A. . . . G. . C. . . . . TC. AA. AT. C. G. . . C. A. . ATT. . C. . . .

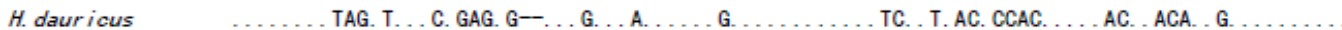

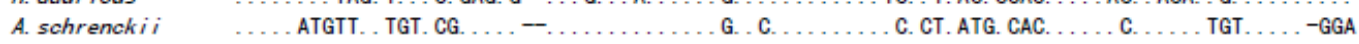
$\begin{array}{rrr}\mid \rightarrow \quad \text { CBS } 2 \quad 720 & \end{array}$

A. baerii G-AGTGTTATCACGAGA-CCTAGTCTTGCCCTCCACCCACATAACAATCAA-GATGCCACAAACGTTTGTTATCGACAAACCCCCTACCC

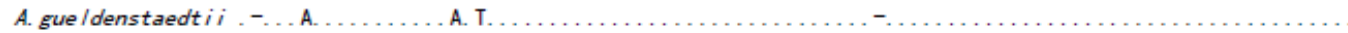

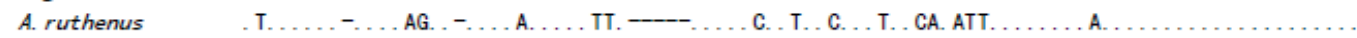

H. dauricus $\quad$ A-G. ... - . . AGA. $-\ldots$ G. T. . . . TAC. . . . G. . . .

A. schrenckii A-GA. ... - . . . AGAG-... G. . T. . AT. T. - . T. . . . . . . G. . -

$\leftarrow$ - $\quad$ I $\quad$ CBS $3 \quad \leftarrow$ -

A. baerii CCTTACGCTGGA-CAAGCCTTATATTTCT TGTCAAACCCCAAAAGCAGGACTGACTTGTCATCAACGTACTCCAATTACCCCCACATGCC

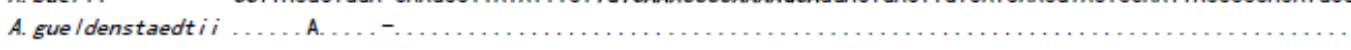

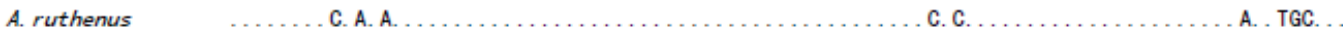

H. dauricus $\quad \ldots$. T. TC. - G. G.

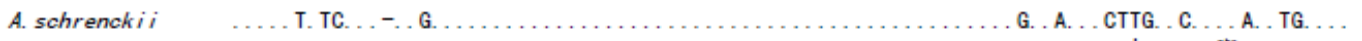

A. baerii TAgCTgtgCAAATATTTATTCACTATATTTTTATGTATATACATTGTTATACAATCACACAAAATAATATATAGCTAGCGTAGCTTAACT

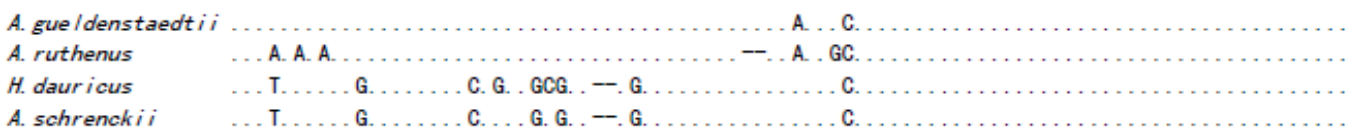

图 25 种鲟鱼tRNA ${ }^{\text {pro }}$, D-loop和tRNAphe序列比对图

Fig. 2 Alignment of sequences of partial tRNA ${ }^{\text {pro }}$, whole D-loop and partial tRNAphe of Acipenser ruthenus, H. huso, A. baerii, A. gueldenstaedtii and A. schrenckii

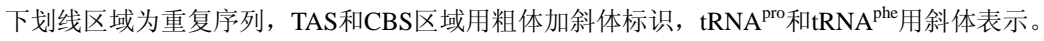

TAS and CBS motifs are in boldface, and repeat units used for calculating secondary structures are underlined and italic, tRNA ${ }^{\text {pro }}$ and tRNA ${ }^{\text {phe }}$ are italic. 
表 15 种鲟鱼样本数、D-loop 全长、AT 含量、重复序列长度、不同重复频率的个体数目

Tab. 1 Species, number of sturgeons, length of D-loop, AT percentage in the D-loop, length of central repeat, and numbers of heteroplasmic sturgeons in relation to frequency as defined by the length variants

\begin{tabular}{|c|c|c|c|c|c|c|c|}
\hline \multirow{2}{*}{ 种类 Species } & \multirow{2}{*}{$\begin{array}{l}\text { 样本数 } \\
\text { Samples }\end{array}$} & \multirow{2}{*}{ 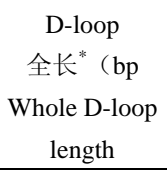 } & \multirow{2}{*}{$\begin{array}{c}\text { D-loop 的 } \\
\text { AT 含量 } \\
\text { AT\% of } \\
\text { Whole D-loop }\end{array}$} & \multirow{2}{*}{$\begin{array}{c}\text { 重复序列长度 } \\
\text { Length of } \\
\text { central repeat } \\
\text { (bp) }\end{array}$} & \multicolumn{3}{|c|}{$\begin{array}{c}\text { 重复片段拷贝数及频率 } \\
\text { Repeat number and frequency (\%) }\end{array}$} \\
\hline & & & & & $\begin{array}{c}4 \text { 拷贝 } \\
\text { Four copies }\end{array}$ & $\begin{array}{c}5 \text { 拷贝 } \\
\text { Five copies }\end{array}$ & $\begin{array}{c}6 \text { 拷贝 } \\
\text { Six copies }\end{array}$ \\
\hline 西伯利亚鲟 Acipenser baerii & 30 & 795 & 62.2 & 82 & 23 & 70 & 7 \\
\hline 俄罗斯鲟 A. gueldenstaedtii & 30 & 796 & 62.5 & 82 & 87 & 23 & 0 \\
\hline 达氏鳇 H. dauricus & 30 & 821 & 62.7 & 78 & 100 & 0 & 0 \\
\hline 小体鲟 A. ruthenus & 30 & 827 & 63 & 80 & 100 & 0 & 0 \\
\hline 史氏鲟 A. schrenckii & 30 & 813 & 61.8 & 82 & 100 & 0 & 0 \\
\hline
\end{tabular}

*全长只包括一个拷贝的重复序列（whole D-loop length only contains one central repeat）。

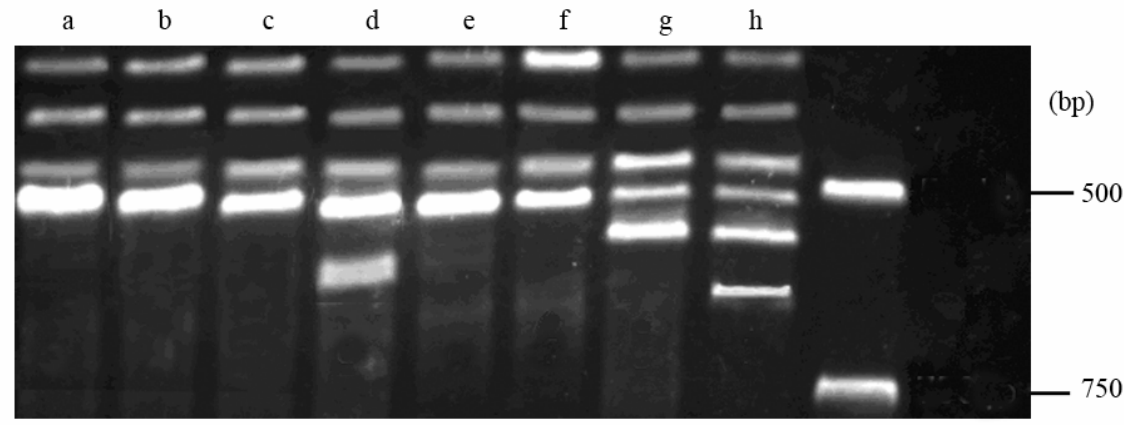

图 35 种鲟鱼 D-loop 的长度差异和多态性电泳图（重复片段拷贝数）

Fig. 3 Resolution of PCR products by agarose gel electrophoresis. Heteroplasmic band pattern (number of repeats)

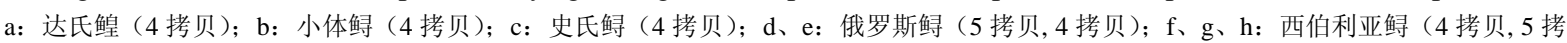
贝, 6 拷贝 )。

a: H. dauricus (4 copies); b: Acipenser ruthenus (4 copies); c: A. schrenckii (4 copies); d, e: A. gueldenstaedtii (5 copies, 4 copies); f, g, h: A. baerii (4 copies, 5 copies, 6 copies).

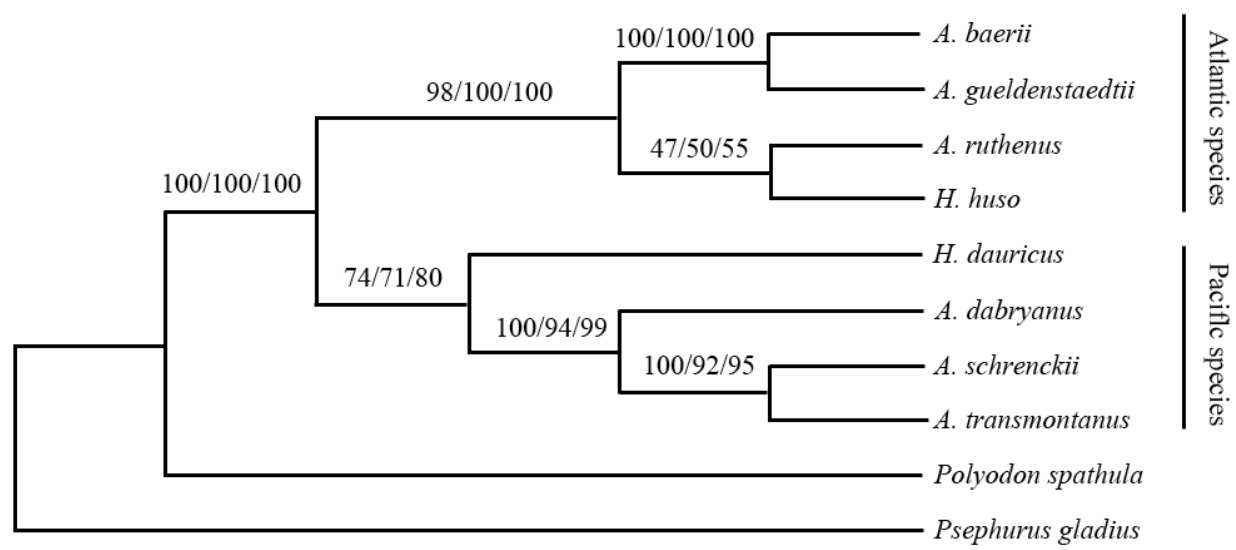

图 4 利用 PAUP 4.0、PHYML 和 MrBayes 构建的鲟鱼最大简约树、最大似然树和贝叶斯树

Fig. 4 Maximum parsimony tree, Maximum likelihood tree and Bayes tree of the sturgeons calculated by PAUP 4.0,

PHYML and MrBayes methods

各自的自展检验值和后验概率（MP/ML/Bayes）分别表示在枝上。

Bootstrap values from 1000 replications and posterior probability are shown on the branches (MP/ML/Bayes).

种分支。

从图 4 的结果明显可以看出达氏鳇和欧洲鳇都
分布在鲟属鱼类之间, 与鲟属互为姊妹种群, 说明 鳇属不太可能是单系群起源。关于鳇鱼的起源问题 
一直存在两个截然不同的观点, 按传统的形态学分 类, 鳇鱼是单系群起源 (Artyukhin, 2006), 但分子 系统学研究结果显示鳇鱼为非单系群起源 (Birstein \& DeSalle, 1998; Ludwig et al, 2001; Ludwig et al, 2000; Zhang et al, 2000), 比如 Zhang et al (2000) 的研究发现达氏鳇与中吻鲟 (Acipenser medirostris) 聚在一起, 而欧洲鳇则处在大西洋种分支中。 Krieger et al（2008）利用线粒体八个基因构建的系 统进化树也表明达氏鳇与中吻鲟和太平洋鲟 (Acipenser mikadoi) 距离较近, 欧洲鳇位于进化 树大西洋种分支中。虽然也有报道利用线粒体多个 基因进行的鲟鱼进化分析结果表明两种鳇鱼聚在 同一分支上, 与小体鲟的距离相近 (Birstein et al, 2002; Birstein \& DeSalle, 1998; Ludwig et al, 2001), 但这可能是由于不同世代和不同地域分布的样本 差异造成的。本研究中鳇属鱼类达氏鳇和欧洲鳇分 布在进化树的两支 (图 4), 分别与一些鲟属互为姊 妹种群, 支持鳇属非单系群起源的观点。

此外, 达氏鳇和欧洲鳇在形态上非常相似, 鳇 鱼与达氏鲟无论在形态还是生态上都有显著差异 (Billard \& Lecointre, 2000), 而且以往的形态学分 类也是把鳇鱼与小体鲟分在不同的类群中 (Artyukhin, 1995; Bemis \& Kynard, 1997), 但是利 用形态学特征和分子遗传标记进行的系统发育研 究都发现两种鳇鱼只有欧洲鳇是与小体鲟聚在一 起 (Artyukhin, 2006; Bemis et al, 1997; Birstein \& DeSalle, 1998), 而且以此两种鱼类为亲本得到的杂 交种也是可育的 (Birstein et al, 1997)。所以目前对 小体鲟为何与鲌鱼进化距离相近尚无结论, 有研究 者推测可能是幼体滞留发育而导致两者进化距离 相近但形态差异大 (Grande et al, 1991)。

\section{2 线粒体 DNA 异质性}

mtDNA 控制区包含重复序列的拷贝数不一样, 反映在电泳上就是条带数不同。只出现 1 条条带的 个体为同质性 (homoplasmic) 个体, 出现 2 条以上 条带的个体为异质性 (heteroplasmic) 个体。本研 究中被检测的 150 个个体全部出现多条条带, 说明 这 5 种鲟鱼的线粒体 DNA 均具异质性, 而且俄罗 斯鲟和西伯利亚鲟出现两种类型的异质性个体, 说 明不同个体间 mtDNA D-loop 包含重复序列的楛贝 数也不一样。迄今为止, 线粒体 DNA 异质性在很 多物种都有发现, 无脊椎动物中的扇贝 (Placopecten magellanicus) (Snyder et al, 1987)、果蝇 (Drosophila melanogaster) (Fauron \& Wolstenholme, 1976)、象 皮虫 (Pissodes nemorensis) (Boyce et al, 1989)、蟋 蟀 (Gryllus firmus) (Harrison et al, 1985), 脊椎动 物, 如两栖类 (Yang et al, 1994)、爬行类 (Densmore et al, 1985; Ray \& Densmore, 2003; Wallis, 1987), 鸟 类 (Mundy et al, 1996)、哺乳类 (Boursot, 1987; Casane et al, 1994; Hayasaka, 1991) 等, 在人类中也 有发现 (Comas et al, 1995)。在鲟鱼以外的鱼类中 已发现 mtDNA 存在异质性特征的有黑鲈 (Dicentrarchus zabrax) (Cesaroni et al, 1997)、美 洲西鲱 (Alosa sapidissima) (Bentzen et al, 1988)、 弓鯺鱼 (Amia calva) (Bermingham et al, 1986)、遮 目鱼 (Chanos chanos) (Ravago et al, 2002)、三斑 圆雀鲷 (Dascyllus trimaculatus) (Chen et al, 2004)、 大西洋鳕 (Gadus morhua) (Arnason \& Rand, 1992)、 小鲤 (Cyprinella spiloptera) (Broughton \& Dowling, 1994)、平鲉 (Sebastes mentella) (Bentzen et al, 1998) 等。

根据已有报道, 27 种鲟形目鱼类中已有 20 种 进行了 mtDNA 异质性研究, 其中有 19 种发现 mtDNA 存在重复序列（包括了两种鳇属鱼类, 一 种铲鲟和十六种鲟属鱼类), 重复片段长度在 74 83 bp 之间, 频率为 1 7 次不等, 最高频率在 2 4 之间, 其中大西洋鲟（Acipenser sturio）和裸腹鲟 (Acipenser nudiventris) 只存在同质性个体，重复 序列频率都为 2 (Ludwig et al, 2000), 白鲟科鱼类 mtDNA 尚未发现重复序列（Brown et al, 1992; Brown et al, 1996; Buroker et al, 1990; Ludwig et al, 2000; Miracle \& Campton, 1995; Zhang et al, 1999)。 本研究第一次报道了达氏鳇和史氏鲟存在 mtDNA 异质性现象。5 种鲟鱼 D-loop 重复序列的最后一个 单元是一个不完全重复单元, 该单元 5' 端部分片段 和其它重复单元一致，但不同种类中一致的片段长 度不一, 至 3' 端开始变异, 变异起始于 TAS3 上游 15 bp 区域内（图 2)。该研究结果和一些其它鲟鱼 的研究结果一致, 例如中华鲟 (Acipenser sinensis), 美洲鲟 (Acipenser oxyrinchus)、白鲟、太平洋鲟、 湖鲟 (Acipenser fulvescens) 中也有类似的情况

(Brown et al, 1996; Zhang et al, 1999)。这说明不完 全重复单元可能是大部分鲟科鱼类线粒体重复序 列 DNA 的共同特征。

本研究中西伯利亚鲟、俄罗斯鲟和小体鲟的重 复序列长度与已报道的一致, 其中西伯利亚鲟重复 
序列的核酸序列与已报道的一致 (Ludwig et al, 2000 ), 但将俄罗斯鲟和小体鲟重复序列的核酸序 列与已报道略有差异, 主要表现在重复序列分成了 相同的两部分, 而且都是从 TAS2 区域分开, 形成 序列相似但排列方式不一样的特点 (图 5), 以此我 们推测不同地域同种鲟鱼的共同祖先的线粒体 DNA 可能发生过分子内重组的现象。此外, 不同种 类的重复序列单元十分相似, 本研究中的达氏鳇和 史氏鲟重复序列单元相似度为 $82.93 \%$, 西伯利亚鲟 和俄罗斯鲟的更是高达 $90.59 \%$, 而且与其它鲟形目 鱼类 D-loop 序列进行比对后发现, 不同种类的鲟鱼 重复序列相似性可达 100\%, 比如西伯利亚鲟与波 斯鲟（Acipenser persicus）（gi:189164316）或俄罗 斯 鲟 (gi:189498273), 俄罗斯鲟与中华鲟 （gi:189164316）或波斯鲟（gi:14422689）等。因 此, 推测重复序列单元的相似性在鲟鱼进化上具有 相关意义, 重复序列在种分化前可能就已经存在, 分子内重组现象可能是在种分化后发生。也有研究
者提出假说认为重复序列和异质性是在种分化后 独立产生的，或者异质性是在种分化前产生的，而 前者得到非正常延伸模型支持 (Miracle \& Campton, 1995)

由于重复片段位于 D-loop 复制的终止区, 有研 究者认为重复序列频率不一的产生是为了形成稳 定的二级结构, 以利于线粒体的单链复制 (Buroker et al, 1990), 而且二级结构的热稳定值 $\triangle \mathrm{G}=-8.0$ $\mathrm{kcal} / \mathrm{mol}$ 是异质性个体和同质性个体的分界线, 异 质性个体 $\triangle \mathrm{G}$ 要小于 $-8.0 \mathrm{kcal} / \mathrm{mol}$, 而同质性个体大 于 $-8.0 \mathrm{kcal} / \mathrm{mol}$ (Ludwig et al, 2000)。从图 6 看本 研究 5 种鲟鱼的 D-loop 重复序列都能形成较稳定的 二级结构, 热稳定值都小于 $-0.8 \mathrm{kcal} / \mathrm{mol}$ 。

造成线粒体 DNA 异质性的原因目前有几种模 型解释：I. 分子间和分子内重组（Rand \& Harrison, 1986 ); II. 滑动错配 (Efstratiadis et al, 1980; Levinson, 1987); III. 非正常延伸或竞争替代模型 (Buroker et al, 1990); IV. 转座（Rand \& Harrison,

$\mid-T H S 2$

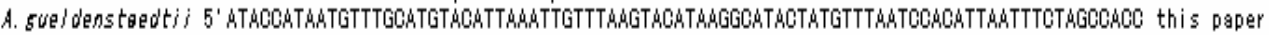

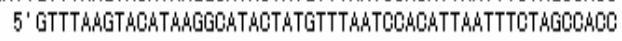

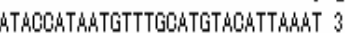
$\mid-T$ TS2- $\mid$ Ludui 5 et al, $(0000)$

A. ruthenus

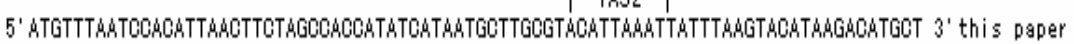

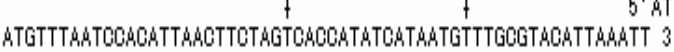

Ludwi e et al, (2000)

图 5 俄罗斯鲟和小体鲟重复序列的比对图

Fig. 5 Alignment of two different central repeat sequences of $A$. gueldenstaedtii and A. ruthenus 变异位点用箭头标示 (TAS2 motifs are marked and mutation sites are arrowed)。
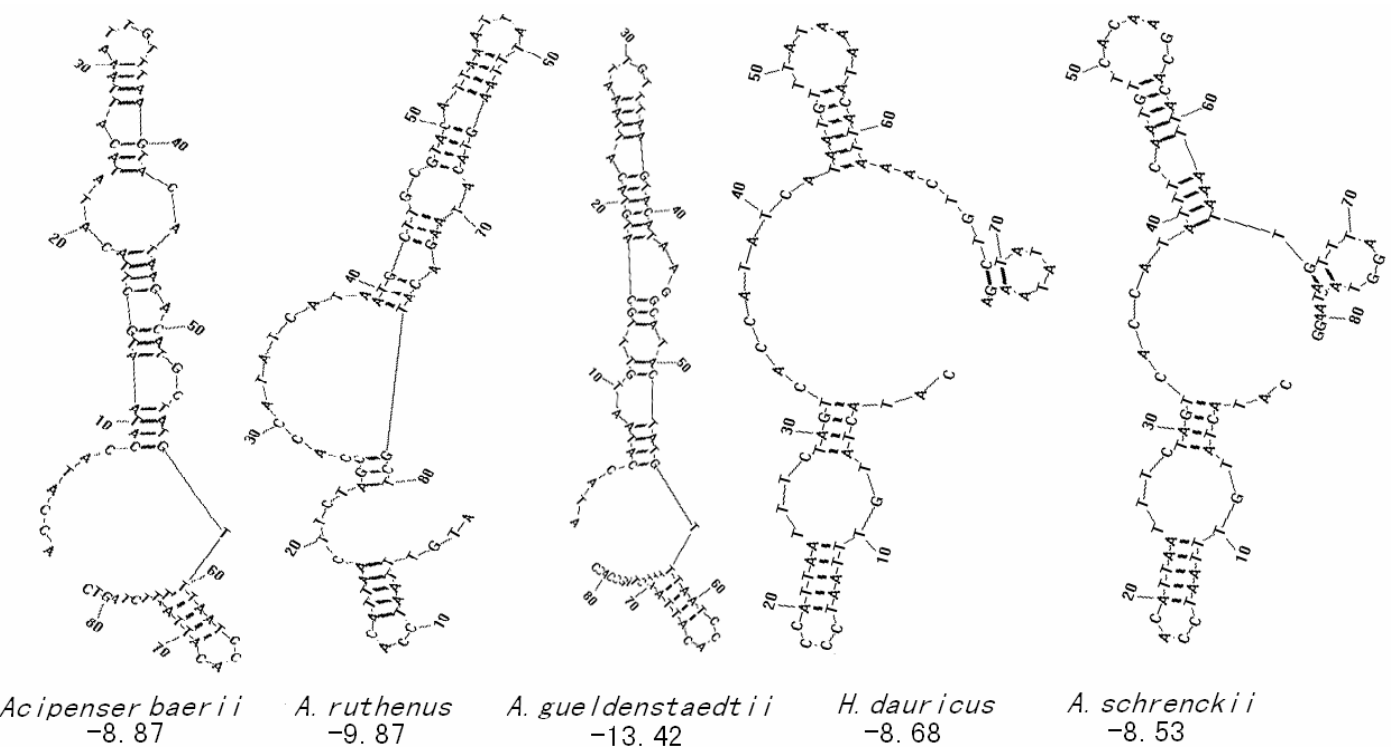

图 6 利用轻链重复序列预测二级结构并计算 $\triangle \mathrm{G}$ 值, $20^{\circ} \mathrm{C}(\mathrm{kcal} / \mathrm{mol})$

Fig. 6 Predicted L-strand secondary structures of central repeat and their $\triangle \mathrm{G}$ values at $20^{\circ} \mathrm{C}(\mathrm{kcal} / \mathrm{mol})$ 
1989); V. H 链和 L 链模型 (Broughton \& Dowling, 1994); VI. TAS 的复制调控模型 (Brown et al, 1996) 等。这些模型提出都是基于各自发现的重复序列和 D-loop 结构提出的, 既有其适用性又有其局限性 (Arnason \& Rand, 1992; Ludwig et al, 2000), 其中 模型 II、III、V 和 VI 都与 mtDNA 复制和调控有关。 目前讨论最多的是非正常延伸模型, 该模型认为重 复序列的增加或减少都是发生在线粒体 DNA H 链 复制时期。合成的新 H 链与 D-loop 双链形成三股 螺旋的动态竞争平衡, 新 $\mathrm{H}$ 链或 D-loop 双链会产 生的发夹结构, 从而导致了 $\mathrm{H}$ 链在继续复制时会增 加或减少重复序列。从目前已报道的研究来看, 鲟 鱼 mtDNA 重复序列的频率多数在 2 3 次, 表明可 能存在对重复序列发生频率的进化选择机制。

Brown et al（1996）提出的 TAS 复制调控模型认为 重复序列频率的高低受到反式作用蛋白因子与 TAS 区域结合的调节, 串联重复会导致 TAS 数目的增 加, 而降低 $\mathrm{H}$ 链的成功复制, 所以高频率的重复序 列会受到负选择调控。Rand (1993) 也认为由于较 长的 mtDNA 分子受到的正选择压力可能会导致插
入稳定的二级结构以利于复制的进行。因此, 重复 序列拷贝的增加或减少可能是正负两种选择机制 的综合结果。

线粒体基因已广泛用于鲟鱼的进化、系统发 育、种群遗传和育种及种质鉴定中，但由于其属于 母系遗传, 所以只能反映母本的遗传特征, 而且由 于鲟鱼种质资源的日益退化，人工培育和野生自然 种群品种的表型与基因型不一致 (Jenneckens et al, 2000; Ludwig et al, 2008) 和基因渐渗的现象

(Arefjev, 1997; Ludwig et al, 2003; Ludwig et al, 2002 ), 这些都表明仅仅依赖线粒体基因作为标记 是不足的。虽然鲟鱼染色体组的多倍性和不同种间 的倍性差异都给核基因分析研究带来困难 (Ludwig et al, 2001)，但发展核基因作为新的遗传标记还是 很需要的（Krieger et al, 2008; Ludwig, 2006)。

致谢: 北京市水产科学研究所十渡鲟鱼养殖基 地的员工在样品采集过程中给予了重要帮助, Ms. Kristin Le Saux-Farmer (0ttawa University) 在 文章英文部分给予了建议, 谨此致以衰心感谢!

\section{参考文献:}

Arefjev VA. 1997. Sturgeons hybrids: Natural reality and practical prospects [J]. Aquacult Mag, 7(8): 52-58.

Arnason E, Rand DM. 1992. Heteroplasmy of short tandem repeats in mitochondrial DNA of Atlantic cod, Gadus morhua [J]. Genetics, 132(1): 211-220.

Artyukhin EN. 2006. Morphological phylogeny of the order Acipenseriformes [J]. J Appl Ichthyol, 22(Suppl. 1): 66-69.

Artyukhin EN. 1995. On biogeography and relationships within the genus Acipenser [J]. Sturgeon Quart, 3(2): 6-8.

Bemis WE, Findeis EK. 1994. The sturgeons' plight [J]. Nature, 370(6491): 602-602.

Bemis WE, Findeis EK, Grande L. 1997. An overview of Acipenseriformes [J]. Environ Biol Fishes, 48(1):

Bemis WE, Kynard B. 1997. Sturgeon rivers: An introduction to acipenseriform biogeography and life history [J]. Environ Biol Fishes, 48(1): 167-183.

Bentzen P, Leggett WC, Brown GG. 1988. Length and restriction site heteroplasmy in the mitochondrial DNA of American shad (Alosa sapidissirna) [J]. Genetics, 118: 509-518.

Bentzen P, Wright JM, Bryden LT, Sargent M, Zwanenburg KCT. 1998. Tandem repeat polymorphism and heteroplasmy in the mitochondrial control region of redfishes (Sebastes: Scorpaenidae) [J]. J Hered, 89(1): 1-7.

Bermingham E, Lamb T, Avise JC. 1986. Size polymorphism and heteropiasmy in the mitochondrial DNA of lower vertebrates [J]. $J$ Hered, 77(4): 249-252.

Billard R, Lecointre G. 2000. Biology and conservation of sturgeon and paddlefish [J]. Rev Fish Biol Fish, 10(4): 355-392.

Birstein VJ, Doukakis P, DeSalle R. 2002. Molecular phylogeny of
Acipenseridae: Nonmonophyly of Scaphirhynchinae [J]. Copeia, 2002(2): 287-301.

Birstein VJ, Hanner R, DeSalle R. 1997. Phylogeny of the Acipenseriformes: Cytogenetic and molecular approaches [J]. Environ Biol Fishes, 48(1): 127-155.

Birstein VJ, DeSalle R. 1998. Molecular phylogeny of Acipenserinae [J]. Mol Phylogen Evol, 9(1): 141-155.

Boursot P. 1987. Heteroplasmy in mice with deletion of a large coding region of mitochondrial DNA [J]. Mol Biol Evol, 4(1): 46-55.

Boyce TM, Zwick ME, Aquadro CF. 1989. Mitochondrial DNA in the bark weevils: Size, structure and heteroplasmy [J]. Genetics, 123(4): 825-836.

Broughton RE, Dowling TE. 1994. Length variation in mitochondrial DNA of the minnow Cyprinella spiloptera [J]. Genetics, 138(1): 179-190.

Brown GG, Gadaleta G, Pepe G, Saccone C, Sbisa E. 1986. Structural conservation and variation in the D-loop-containing region of vertebrate mitochondrial DNA [J]. J Mol Biol, 192(3): 503-511.

Brown JR, Beckenbach AT, Smith MJ. 1992. Mitochondrial DNA length variation and heteroplasmy in populations of white sturgeon (Acipenser transmontanus) [J]. Genetics, 132(1): 221-228.

Brown JR, Beckenbach K, Beckenbach AT, Smith MJ. 1996. Length variation, heteroplasmy and sequence divergence in the mitochondrial DNA of four species of sturgeon (Acipenser) [J]. Genetics, 142(2): 525-535.

Brown WM. 1985. The mitochondrial genome of animals [M]//RJ M, editor. Molecular Evolutionary Genetics: Monographs in Evolutionary Biology. New York: Plenum Publishing Corporation, 95-130.

Brown WM, George M, Wilson AC. 1979. Rapid evolution of animal mitochondrial DNA [J]. Proc Natl Acad Sci USA, 76(4): 1967-1971. 
Buroker NE, Brown JR, Gilbert TA, O'Hara PJ, Beckenbach AT, Thomas WK, Smith MJ. 1990. Length heteroplasmy of sturgeon mitochondrial DNA: An illegitimate elongation model [J]. Genetics, 124(1): 157-163.

Casane D, Dennebouy N, De-Rochambeau H, Mounolou JC, Monnerot M. 1994. Genetic analysis of systematic mitochondrial heteroplasmy in rabbits [J]. Genetics, 138(2): 471-480.

Cesaroni D, Venanzetti F, Allegrucci G, Sbordoni V. 1997. Mitochondrial DNA length variation and heteroplasmy in natural populations of the European sea bass, Dicentrarchus labrax [J]. Mol Biol Evol, 14(5): 560-568.

Chen CA, Ablan MCA, McManus JW, Bell JD, Tuan VS, Cabanban AS, Shao KT. 2004. Variable numbers of tandem repeats (VNTRs), heteroplasmy, and sequence variation of the mitochondrial control region in the three-spot Dascyllus, Dascyllus trimaculatus (Perciformes: Pomacentridae) [J]. Zool Stud, 43(4): 803-812.

Clayton DA. 1982. Replication of animal mitochondrial DNA [J]. Cell, 28(4): 693-705.

Clayton DA. 1984. Transcription of the mammalian mitochondrial genome [J]. Annu Rev Biochem, 53(1): 573-594.

Comas D, Pääbo S, Bertranpetit J. 1995. Heteroplasmy in the control region of human mitochondrial DNA [J]. Genome Res, 5(1): 89-90.

Densmore LD, Wright JW, Brown WM. 1985. Length variation and heteroplasmy are frequent in mitochondrial DNA from parthenogenetic and bisexual lizards (genus Cnemidophorus) [J]. Genetics, 110(4): 689-707.

Efstratiadis A, Posakony JW, Maniatis T, Lawn RM, O'Connell C, Spritz RA, DeRiel JK, Forget BG, Weissman SM, Slightom JL. 1980. The structure and evolution of the human $\beta$-globin gene family [J]. Cell, 21(3): 653-668.

Fauron CMR, Wolstenholme DR. 1976. Structural heterogeneity of mitochondrial DNA molecules within the genus Drosophila [J]. Proc Natl Acad Sci USA, 73(10): 3623-3627.

Felsenstein J. 1985. Confidence limits on phylogenies: An approach using the bootstrap [J]. Evolution, 39(4) : 783-791.

Gardiner BG. 1984. Sturgeons as living fossils [M]//Eldredge N SSe, editor. Living Fossils. New York: Springer Verlag Press, 148-152.

Grande L, Bemis W, Paleontology SoV. 1991. Osteology and phylogenetic relationships of fossil and recent paddle fishes (Polyodontidae) with comments on the interrelationships of Acipenseriformes [J]. $J$ Vertebr Paleontol, 11(Suppl. 1): 1-121.

Guindon S, Gascuel O. 2003. A simple, fast, and accurate algorithm to estimate large phylogenies by maximum likelihood [J]. Syst Biol, 52(5): 696-704.

Harrison RG, Rand DM, Wheeler WC. 1985. Mitochondrial DNA size variation within individual crickets [J]. Science, 228(4706): 1446-1448.

Hauswirth WW, Clayton DA. 1985. Length heterogeneity of a conserved displacement-loop sequence in human mitochondrial DNA [J]. Nucleic Acids Res, 13(22): 8093-8104.

Hayasaka K. 1991. Heteroplasmy and polymorphism in the major noncoding region of mitochondrial DNA in Japanese monkeys: Association with tandemly repeated sequences [J]. Mol Biol Evol, 8(4): 399-415.

Jenneckens I, Meyer JN, Debus L, Pitra C, Ludwig A. 2000. Evidence of mitochondrial DNA clones of Siberian sturgeon, Acipenser baerii, within Russian sturgeon, Acipenser gueldenstaedtii, caught in the River Volga [J]. Ecol Lett, 3(6): 503-508.

Krieger J, Hett AK, Fuerst PA, Artyukhin E, Ludwig A. 2008. The molecular phylogeny of the order Acipenseriformes revisited [J]. $J$ Appl Ichthyol, 24(s1): 36-45.

Lee WJ, Conroy J, Howell WH, Kocher TD. 1995. Structure and evolution of teleost mitochondrial control regions [J]. J Mol Evol, 41(1): 54-66.
Levinson G. 1987. Slipped-strand mispairing: A major mechanism for DNA sequence evolution [J]. Mol Biol Evol, 4(3): 203-221.

Ludwig A. 2006. A sturgeon view on conservation genetics [J]. Eur J Wildl Res, 52(1): 3-8.

Ludwig A, Arndt U, Lippold S, Benecke N, Debus L, King T, Matsumura S. 2008. Tracing the first steps of American sturgeon pioneers in Europe [J]. BMC Evol Biol, 8(1): 221-252.

Ludwig A, Belfiore NM, Pitra C, Svirsky V, Jenneckens I. 2001. Genome duplication events and functional reduction of ploidy levels in sturgeon (Acipenser, Huso and Scaphirhynchus) [J]. Genetics, 158(3): 1203-1215.

Ludwig A, Congiu L, Pitra C, Fickel J, Gessner J, Fontana F, Patarnello T, Zane L. 2003. Nonconcordant evolutionary history of maternal and paternal lineages in Adriatic sturgeon [J]. Mol Ecol, 12(12): 3253-3264.

Ludwig A, Debus L, Jenneckens I. 2002. A molecular approach for trading control of black caviar [J]. Int Rev Hydrobiol, 87: 661-674.

Ludwig A, May B, Debus L, Jenneckens I. 2000. Heteroplasmy in the mtDNA control region of sturgeon (Acipenser, Huso and Scaphirhynchus) [J]. Genetics, 156(4): 1933-1947.

Miracle AL, Campton DE. 1995. Tandem repeat sequence variation and length heteroplasmy in the mitochondrial DNA D-loop of the threatened Gulf of Mexico sturgeon, Acipenser oxyrhynchus desotoi [J]. $J$ Hered, 86(1):2 2-27.

Mundy NI, Winchell CS, Woodruff DS. 1996. Tandem repeats and heteroplasmy in the mitochondrial DNA control region of the loggerhead shrike (Lanius ludovicianus) [J]. J Hered, 87(1): 21-26.

Nylander JAA. 2004. MrModeltest v2[Z]. Program distributed by the author. Evolutionary Biology Centre, Uppsala University.

Quattro J, Greig T, Coykendall D, Bowen B, Baldwin J. 2002. Genetic issues in aquatic species management: the shortnose sturgeon (Acipenser brevirostrum) in the southeastern United States [J]. Conserv Genet, 3(2): 155-166.

Posada D, Crandall KA. 1998. MODELTEST: Testing the model of DNA substitution [J]. Bioinformatics, 14(9): 817-818.

Rand DM, Harrison RG. 1986. Mitochondrial DNA transmission genetics in crickets [J]. Genetics, 114(3): 955-970.

Rand DM, Harrison RG. 1989. Molecular population genetics of mtDNA size variation in crickets [J]. Genetics, 121(3): 551-569.

Ravago RG, Monje VD, Juinio-Menez MA. 2002. Length and sequence variability in mitochondrial control region of the milkfish, Chanos chanos [J]. Mar Biotechnol : NY, 4(1): 40-50.

Ray DA, Densmore LD. 2003. Repetitive sequences in the crocodilian mitochondrial control region: Poly-A sequences and heteroplasmic tandem repeats [J]. Mol Biol Evol, 20(6): 1006-1013.

Ronquist F, Huelsenbeck JP. 2003. MrBayes 3: Bayesian phylogenetic inference under mixed models [J]. Bioinformatics, 19(12): 1572-1574.

Snyder M, Fraser AR, LaRoche J, Gartner-Kepkay KE, Zouros E. 1987. Atypical mitochondrial DNA from the deep-sea scallop Placopecten magellanicus [J]. Proc Natl Acad Sci USA, 84(21): 7595-7599.

Swofford DL. 1998. PAUP*: Phylogenetic Analysis Using Parsimony (* and Other Methods):: Version 4[M]. Sunderland, MA. Massachusetts: Sinauer Associates.

Thompson JD, Gibson TJ, Plewniak F, Jeanmougin F, Higgins DG. 1997. The CLUSTAL_X windows interface: Flexible strategies for multiple sequence alignment aided by quality analysis tools [J]. Nucleic Acids Res, 25(24): 4876-4882.

Walberg MW, Clayton DA. 1981. Sequence and properties of the human KB cell and mouse L cell D-loop regions of mitochondrial DNA [J]. Nucleic Acids Res, 9(20): 5411.

Wallis GP. 1987. Mitochondrial DNA insertion polymorphism and germ line heteroplasmy in the Triturus cristatus complex [J]. Heredity, $\mathbf{5 8}$ 
229-238.

Walter A, Turner D, Kim J, Lyttle M, Muller P, Mathews D, Zuker M. 1994. Coaxial stacking of helixes enhances binding of oligoribonucleotides and improves predictions of RNA folding [J]. Proc Natl Acad Sci USA, 91(20): 9218-9222.

Yang YJ, Lin YS, Wu JL, Hui CF. 1994. Variation in mitochondrial DNA and population structure of the Taipei treefrog Rhacophorus taipeianus in Taiwan [J]. Mol Ecol Notes, 3(3): 219-228.

Zhang SM, Deng H, Wang DQ, Zhang YP, Wu QJ. 1999. Mitochondrial DNA length variation and heteroplasmy in Chinese sturgeon (Acipenser sinensis) [J]. Acta genet Sin, 26(5): 489-496. [张四明, 邓 怀, 汪登强, 张亚平, 吴清江. 1999. 中华鲟(Acipenser sinensis)间的
长度变异与个体内的长度异质性. 遗传学报, 26 (5): 489 -496.]

Zhang SM, Zhang YP, Zhang XZ, Chen YJ, Deng H, Wang DQ, Wei QW, Zhang YW, Nie L, Wu QJ. 2000. Molecular phylogenetic systematics of twelve species of Acipenseriformes based on mtDNA ND4L-ND4 gene sequence analysis [J]. Sci Chn: Ser C-Life Sci, 43(2): 129-137.

Zhang SM, Wang DQ, Zhang YP. 2003. Mitochondrial DNA variation, effective female population size and population history of the endangered Chinese sturgeon, Acipenser sinensis [J]. Conserv Genet, 4(6): 673-683.

Zuker M. 1989. On finding all suboptimal foldings of an RNA molecule [J]. Science, 244(4900): 48-52.

\section{东北农业大学畜牧学科简介}

东北农业大学畜牧学科于 1995 年设立博士后流动站, 2000 年被批准为具有博士学位授 予权的一级学科点。本学科有数量遗传与动物育种、分子遗传与动物育种、动物生殖生理与 胚胎工程、动物生物信息学 4 个稳定的研究方向。现有教职员工 30 人，其中教授 7 人 (含相 当职称人员)，副教授 5 人，讲师 14 人 (含相当职称人员)，助教 4 人 (含相当职称人员); 博士生导师 5 人，硕士生导师 10 人。“十一・五”期间学科成员主持承担国家 973 项目子课 题、国家自然科学基金项目、农业部现代农业生物技术体系建设项目、国家公益性行业科研 专项子课题、黑龙江省自然科学基金重点项目等 25 项科研项目。科研成果获国家科技进步二 等奖 1 项，黑龙江省科技进步二等奖 1 项; 发表 SCI 收录论文 24 篇; 出版教材专著 5 部; 3 项发明专利获得授权。

二级学科动物遗传育种与繁殖学科始建于 1948 年，1978 年开始招收硕士研究生，1981 年建立硕士点，1986 年设立博士点，1989 年被评为省重点学科，1999 年被评为农业部重点 学科, 2007 年被评为国家重点 (培育) 学科, 是东北农业大学 “211 工程”重点建设学科。

\section{杨秀芹}

